\title{
BUBBLE EXPANSION COMPUTATIONS AT LOW-MACH NUMBER REGIME
}

\author{
Alberto BECCANTINI ${ }^{1}$, Cong-Huan PHAN ${ }^{1,2}$, Christophe CORRE $^{2}$ \\ ${ }^{1}$ Université Paris-Saclay, CEA, Service d'études mécaniques et thermiques \\ 91191 Gif-sur-Yvette, France \\ cong-huan.phan@cea.fr, alberto.beccantini@cea.fr \\ ${ }^{2}$ Ecole Centrale de Lyon, LMFA UMR 5509 \\ 69134 Ecully, France \\ cong-huan.phan@ec-lyon.fr, christophe.corre@ec-lyon.fr
}

Key words: bubble expansion, low-Mach number flows, artificial compressibility method, exergy criterion

\begin{abstract}
A 1D bubble expansion problem is solved using standard compressible methods whose numerical solutions are compared with the exact low-Mach solution of the problem. The low Mach solution is proved to provide a very good approximation of the grid-converged compressible solution for a weak expansion and an envelope solution for the strong expansion. This motivates the design of a newly proposed Artificial Compressibility solver which will speed up low-Mach simulations of interest for nuclear safety with respect to standard compressible solvers.
\end{abstract}

\section{INTRODUCTION}

High pressure bubble expansion in liquid is involved in several safety problems studied in the Nuclear Industry, such as Borax-type accidents in pool-type experimental reactors or Hypothetical Core Disruptive Accident (HCDA) in a liquid-sodium fast breeder reactor. A Borax-type accident involves a highpressure bubble created by the interaction of melted metal materials with water. For a liquid-sodium fast breeder reactor, the scenario of an HCDA supposes the reactor core has partially melted and the molten fuel chemically interacts with the liquid sodium to produce a large quantity of gaseous components [1]. The explosive expansion of the high-pressure gas bubble formed in the core centre yields significant loads on the reactor vessel. The MARA experimental program was carried out by CEA in the 1980s on a 1/30-scale model of the Superphenix reactor in order to provide reference measurements (pressure at transducers, structure deformation) to be used for validation of the codes applied to the HCDA fluidstructure interaction problem. In the experiments, water replaces the liquid sodium cooling the reactor core and the cover gas below the reactor roof is air instead of argon; the explosion is triggered by an explosive charge. The MARA program involved 10 tests of increasing complexity with additional internal deformable structures gradually accounted for : from MARA 1 and 2 considering a plain vessel with a rigid roof [2] to MARA 10 including the core support structures and the above core structures [3] (see Fig.1).

Measurements provided by the MARA experiments have been especially useful to assess the perfor- 

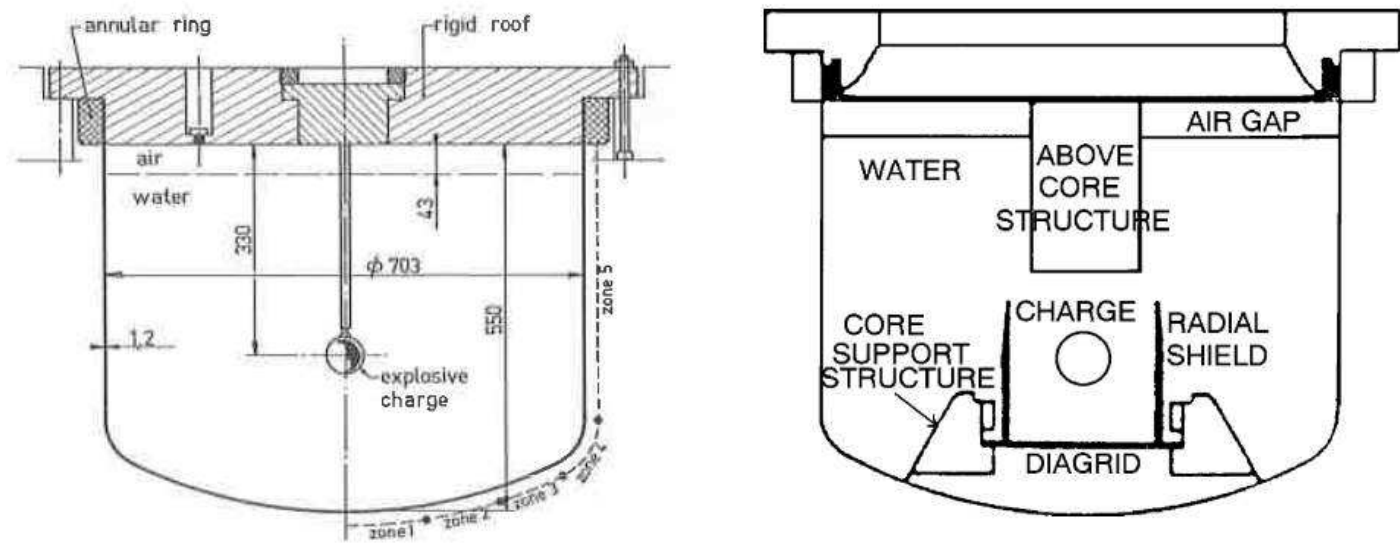

Figure 1: Left : schematic view of the MARA 1/2 configuration taken from [4]. The charge creating the gas bubble is located at the center of the liquid domain; the layer of air below the vessel cover is also visible. Right schematic view of the MARA 10 configuration taken from [5] displaying the added structures included in the fluid-structure interaction.

mance of fluid-structure coupling strategies developed in various codes (SIRIUS, CASTEM-PLEXUS, EUROPLEXUS), see [6] [7]. The present work is focused on a different numerical issue which arises when computing an HCDA-type bubble expansion problem. Two speed scales are associated with the flow field evolution: the sound speed $c$ and the flow speed $u=M c$ with a local Mach number $M$ which can be much lower than problems for standard expansion: an accuracy numerical diffusion on the one hand, scaling like with amplitude $\rho c \Delta u$. The efficiency problem resu
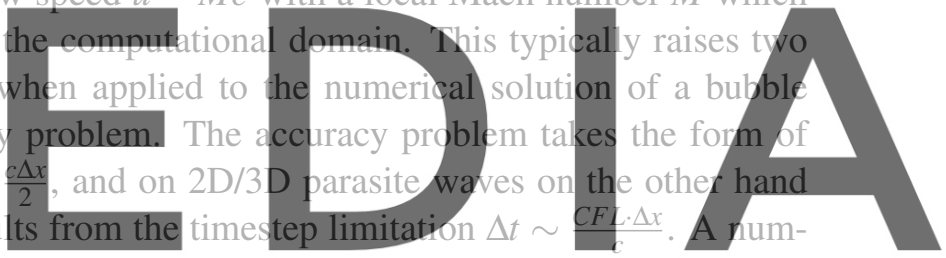

ber of numerical fixes have been proposed in the literature to address these issues for compressible CFD

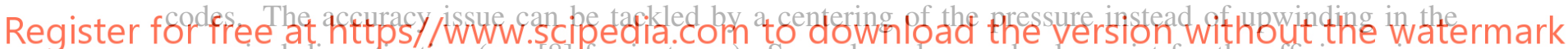
numerical discretization (see [8] for instance). Several workarounds also exist for the efficiency issue

such as the implicit Roe-Turkel approach (though it proves not so efficient in closed geometries such as the ones encountered for HCDA) or the numerical reduction of the sound speed (though it yields incorrect isentropic transformations). In the present work, a new fix is proposed which relies on artificial compressibility (AC), with lower sound speed and low Mach correction for (correct) isentropic transformations. The relevance of the proposed strategy will be demonstrated for 1D low-Mach bubble expansion problems, modelling an HCDA configuration. More precisely, we wish to establish that the efficiency gain offered by AC is not obtained at the expense of a lack of accuracy. The idea in this first stage is to assess whether the exact low-Mach solution of the 1D bubble expansion problem represents a relevant approximation with respect to the fully compressible numerical solutions of the same problem. If the comparison is conclusive, it will motivate the subsequent development of a numerical solver based on the low-Mach model. Due to the length constraints of the present paper, only the key underlying principles of the proposed approach are briefly presented in section 2 - more details will be provided in [9]. The 1D bubble expansion problem is described in section 3. The derivation of the low-Mach number reference solution for this model problem is reviewed and an accuracy criterion based on exergy is proposed. Section 4 is devoted to the comparison of the low-Mach reference solution with fully com- 
pressible simulations performed on a series of increasingly refined grids. The comparison is performed for two bubble expansion problems corresponding to two different initial pressure levels in the bubble region; this allows to better assess the range of validity of the low-Mach model.

\section{NUMERICAL APPROACH}

The newly proposed Artificial Compressibility (AC) numerical approach relies on the following splitting of the Euler equations governing the flow evolution :

$$
\begin{aligned}
& \frac{\partial \rho}{\partial t}+\vec{v} \cdot \vec{\nabla} \rho \quad=\quad 0 \quad-\rho \vec{\nabla} \cdot \vec{v} \\
& \frac{\partial \vec{v}}{\partial t}+(\vec{v} \cdot \vec{\nabla}) \vec{v}+\frac{1}{\rho} \vec{\nabla} P=\quad 0 \\
& \frac{\partial P}{\partial t}+\vec{v} \cdot \vec{\nabla} P \quad=\quad-\rho c_{\mathrm{AC}}^{2} \vec{\nabla} \cdot \vec{v} \quad-\rho\left(c^{2}-c_{\mathrm{AC}}^{2}\right) \vec{\nabla} \cdot \vec{v} \\
& \text { part I } \\
& \text { part II }
\end{aligned}
$$

Part I in the above system corresponds to the convective mechanism with fast acoustic waves filtered through the use of a (numerical) AC sound speed $c_{\mathrm{AC}}$ lower or even much lower than the physical sound speed $c$. Part II includes the contribution of the homogeneous term $(\vec{\nabla} \cdot \vec{v})$, responsible for the isentropic transformations of density and pressure, which takes into account the filtered acoustic waves.

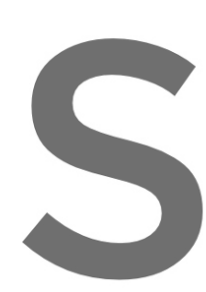
The key ingredients of the of an HCDA problem su Discrete Equation Methor method schematically on primitive variables for for time explieit integration, iv) a
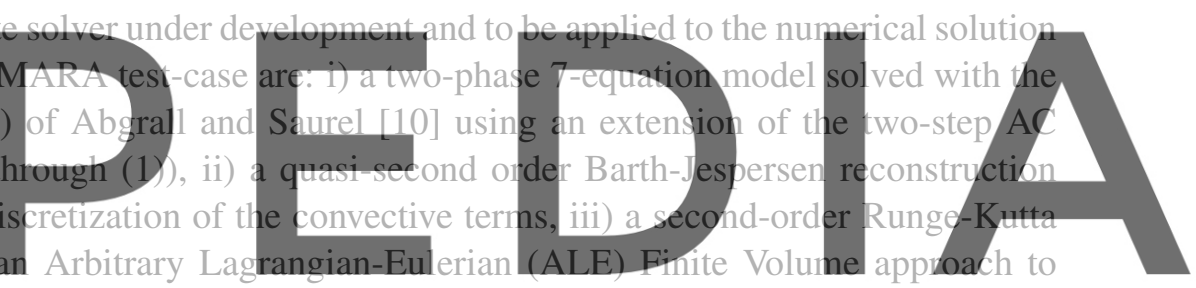
take into account the fluid-structure interaction (the structure surrounding the fluid being solved with

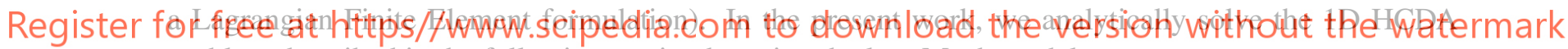
problem described in the following section by using the low-Mach model.

\section{1D BUBBLE EXPANSION PROBLEM}

\subsection{Presentation of the problem}

The proposed 1D bubble expansion problem includes the key features of the full HCDA problem investigated through the MARA experiments. The 1D domain is divided into 3 regions (see Fig. 2) representative of the regions appearing in the MARA experiment : region 1 (or B for Bubble) corresponds to the high pressure gas bubble, region 2 (or $\mathrm{W}$ for water) corresponds to the surrounding water and region 3 (or A for Absorber) corresponds to the low pressure cover gas. In the present simplified analysis, the $1 \mathrm{D}$ domain is assumed rigid, of total length $12 \mathrm{~m}$. At $t=0$, the respective lengths of the sub-regions are $l_{B}=l_{A}=3 \mathrm{~m}, l_{W}=6 \mathrm{~m}$.

Both $\mathrm{B}$ and $\mathrm{A}$ are assumed to behave as perfect gases (with $\gamma=1.4$ ) while water is described by the stiffened gas equation of state, with $\gamma=4.4, p_{\infty}=6.0 \times 10^{8}$ ); its sound speed is equal to $\approx 1600 \mathrm{~m} / \mathrm{s}$. The ideal solution of the problem is such that water behaves like a rigid body oscillating around an equilibrium position. 


\begin{tabular}{|l|c|c|}
\hline $\begin{array}{l}\text { High pressure } \\
\text { gas bubble, } 1\end{array}$ & Water, 2 & $\begin{array}{c}\text { Low pressure } \\
\text { gas, } 3\end{array}$ \\
\hline
\end{tabular}

Figure 2: Flow regions for the 1D HCDA problem.

\begin{tabular}{|c|c|c|c|}
\hline & Region 1 (B) & Region 2 $(\mathrm{W})$ & Region 3 $(\mathrm{A})$ \\
\hline$\rho\left(\mathrm{kg} / \mathrm{m}^{3}\right)$ & 26.827 & 1000.0 & 1.0 \\
$P(\mathrm{~Pa})$ & $1 \times 10^{7}$ & $1 \times 10^{5}$ & $1 \times 10^{5}$ \\
$u(\mathrm{~m} / \mathrm{s})$ & 0.0 & 0.0 & 0.0 \\
\hline
\end{tabular}

Table 1: Initial conditions for the strong bubble expansion.

The initial states in regions B, W and A are summarized in Tables 1 and 2. The two configurations under study differ by the initial pressure level in the bubble, respectively 100 or 10 bars, yielding a strong or weak expansion of the bubble.

\subsection{Low-Mach number reference solution}

In the compressible gases, we have to determine $\rho(x, t), u(x, t), p^{\prime}(x, t)$ and $P(t)\left(p^{\prime}<<P\right)$ such that
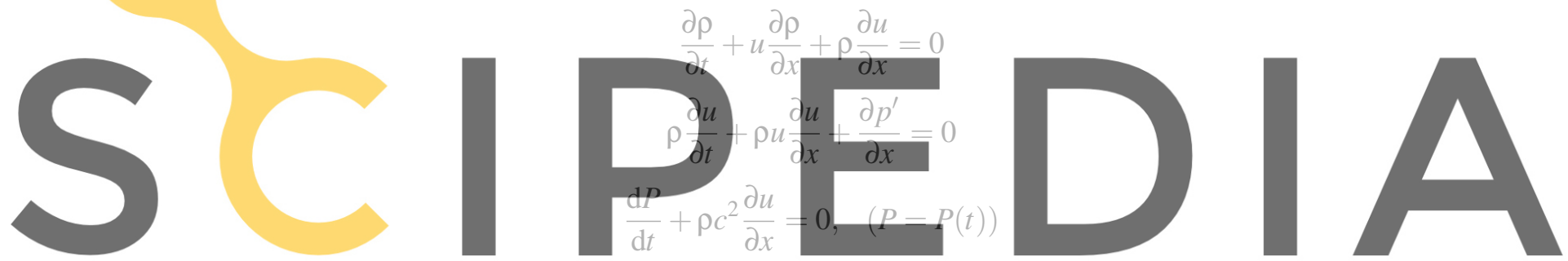

In the incompressible liquid, we have to determine $u_{W}(x, t), p_{W}(x, t)$ such that

Register for free at https//www.scipediancom to down load the version without the watermark

$$
\begin{gathered}
\rho_{W} \frac{u_{W}}{\partial t}+\rho_{W} u_{W} \frac{\partial x_{W}}{\partial x}+\frac{\partial q_{W}}{\partial x}=0 \\
\frac{\partial u_{W}}{\partial x}=0
\end{gathered}
$$

For the incompressible liquid water, the flow speed $u_{W}(t)$ is homogeneous and the pressure is linearly varying between $P_{B}(t)$ and $\left.P_{A}(t)\right)$. For the compressible gases in regions $\mathrm{A}$ and $\mathrm{B}$ the thermodynamic pressure $P(t)$ follows the isentropic law while the velocity is linearly varying in the gases between 0 and $u_{W}$. Mass is conserved and density also satisfies the isentropic law. The low-Mach solution is eventually obtained by integrating in time the ODE describing the motion of the liquid column.

\begin{tabular}{|c|c|c|c|}
\hline & Region 1 (B) & Region 2 $(\mathrm{W})$ & Region 3 $(\mathrm{A})$ \\
\hline$\rho\left(\mathrm{kg} / \mathrm{m}^{3}\right)$ & 5.1795 & 1000.0 & 1.0 \\
$P(\mathrm{~Pa})$ & $1 \times 10^{6}$ & $1 \times 10^{5}$ & $1 \times 10^{5}$ \\
$u(\mathrm{~m} / \mathrm{s})$ & 0.0 & 0.0 & 0.0 \\
\hline
\end{tabular}

Table 2: Initial conditions for the weak bubble expansion. 


\subsection{Exergy as accuracy criterion}

When conservative numerical methods are used to solve compressible flows, total energy conservation cannot be used as a criterion of solution accuracy since automatically satisfied. Numerical entropy production could be retained as a better criterion, however entropy is in $(\mathrm{J} / \mathrm{K})$ and we would rather monitor a physical quantity homogeneous with energy. Because of entropy production, part of the initial energy is not transformed into kinetic energy for the liquid but numerically converted into heat, reducing the so-called "exergy" $(\mathrm{J})$, that is "the amount of work obtainable when some matter is brought to a state of thermodynamic equilibrium with the common components of the natural surroundings by means of reversible processes" [11]. For the 1D HCDA test-case under study, exergy is also the maximum amount of energy which, at any time, can be converted into kinetic energy (of the liquid). Anergy (J) is equal to the initial energy minus the exergy. From an energetic point of view, the following identity holds :

$$
\underbrace{\frac{1}{\gamma-1} \int_{\mathrm{V}_{\mathrm{B}}} P \mathrm{~d} V}_{\mathrm{E}_{\mathrm{B}}}+\underbrace{\frac{1}{\gamma-1} \int_{\mathrm{V}_{\mathrm{A}}} P \mathrm{~d} V}_{\mathrm{E}_{\mathrm{A}}}+\underbrace{\frac{1}{2} \int_{\mathrm{V}_{\mathrm{W}}} \rho v^{2} \mathrm{~d} V}_{\mathrm{K}_{\mathrm{W}}}+E_{W}=\text { constant }
$$

where $E_{W}$ is the internal energy of the liquid. According to the motion equation and assuming the variation of $E_{W}$ remains negligible, the velocity (and thus the kinetic energy) of the liquid is maximum when the acceleration is zero, i.e. when both $P_{B}$ and $P_{A}$ are equal to the "equilibrium" pressure $P_{e}$. As the anergy (and the entropy), $P_{e}$ will increase in time if dissipative phenomena take place in the flow. Without (physical) dissipative phenomena occurring in the flow, the more accurate the numerical discretization is, the lower the value of $P_{e}$ remains. From the time e
$P_{e}$ and $V_{A, e}$ at each time by writing :
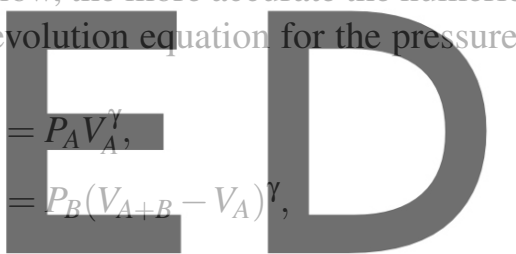

where $V_{A+B}$ is the total volume of the gas (constant in time). For the case of the low-Mach solution of

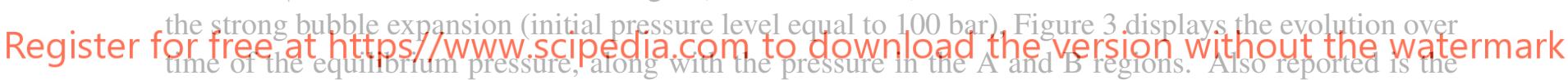
evolution of exergy, total energy, liquid kinetic energy and total energy in regions A and B. Regarding the pressure evolution, it can be noticed the decrease of the bubble pressure corresponds, because of the motion of the liquid column, to an increase of the absorber pressure; the equilibrium pressure remains constant over time for the low-Mach reference solution (no dissipative phenomena). It will be monitored for the compressible calculation and used as an accuracy criterion. Regarding the energy evolution, it can be noticed that exergy is also constant over time, as expected, and equal to the maximum of the water kinetic energy.

\section{Numerical investigation: Compressible solutions vs reference low-Mach number solution}

\subsection{Numerical method for compressible solutions}

Compressible solutions are computed using the Discrete Equation Method (DEM) for two-phase flow with the HLLC solver, including Barth-Jespersen second-order reconstruction on primitive variables; time-integration is performed using a second-order Runge-Kutta approach. An instantaneous relaxation of the pressure and of the velocity is also applied to prevent negative pressure in the water. In order to 


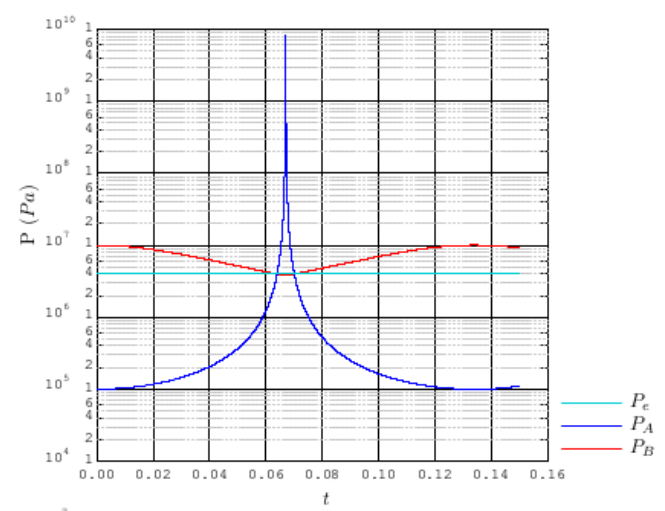

$\times 10^{7}$

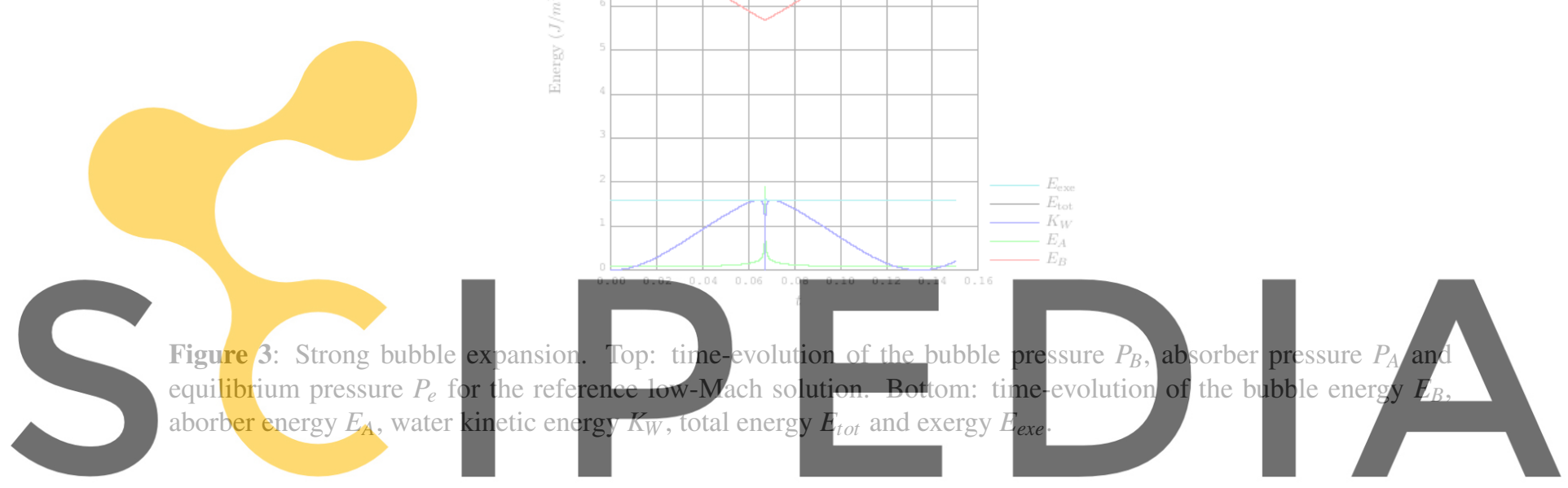

ensure a reliable comparison between the compressible solutions and the quasi-analytically computed

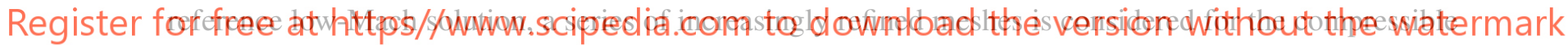
simulation: 192 cells (coarse), 384 cells (medium), 768 cells (fine). In the strong expansion case, the maximum speed of the liquid is about $70 \mathrm{~m} / \mathrm{s}$, which remains lower than sound speeds for liquid and gases.

\subsection{Strong bubble expansion}

Figures 4 to 6 display the pressure and the velocity distributions computed on the coarse, medium and fine grids using the fully compressible model and compare them with the low-Mach solution at selected times $t=4 \mathrm{~ms}, t=42 \mathrm{~ms}$ and $t=68 \mathrm{~ms}$. In the early stage of the bubble expansion $(t=4 \mathrm{~ms}$, see Fig. 4) it can be observed how the liquid column is accelerated by the acoustic waves propagating in the closed domain. At a later time, $t=42 \mathrm{~ms}$, it can be observed in Fig. 5 how the compressible solutions are much closer to the low-Mach number solution. Time $t=68 \mathrm{~ms}$ (see Fig. 6) corresponds to the impact of the liquid column with the highly compressed absorber gas region $\mathrm{A}$ at the right end of the flow domain. The large differences observed between the compressible solutions and the reference lowMach solution, be it on the pressure or on the velocity, are actually produced by the time-shift between the compressible solutions where the acoustic waves propagate in the water with a finite velocity and 
the low-Mach solution where this propagation takes place at an infinite speed. This is clearly observed in Fig. 7 where the time-evolution of the average pressure in the bubble and in the absorber region is displayed for both the low-Mach reference solution and the compressible solution computed on the coarse, medium and fine grids. Time $t=68 \mathrm{~ms}$ corresponds to the time where the bubble average pressure reaches its minimum value, the absorber reaching its maximum value at the same time. Because of the finite speed of propagation for the acoustic waves in the compressible calculation, the minimum bubble pressure or absorber peak pressure is reached slightly later (hence the large pressure difference previously observed in Fig. 6). Another consequence is the much larger peak of pressure observed in the absorber for the low-Mach solution with respect to the compressible solution. The finite value of the sound speed is indeed responsible for the weaker momentum variation of the liquid column in the compressible solutions. The plot of the time-evolution for the equilibrium pressure displayed in Fig. 8 shows the equilibrium pressure decreases with the mesh size for the compressible numerical solution. However, it does not exactly converge to its initial value because of the entropy/anergy production at the impact of the liquid column on the right end of the closed domain. Keeping in mind that HCDA analysis is particularly focused on the structural loads induced by the bubble expansion, it is worth noticing that low-Mach number and compressible solutions remain close and that the low-Mach number solution provides a useful upper limit for the compressible simulations as far as pressure and kinetic energy of water are concerned.
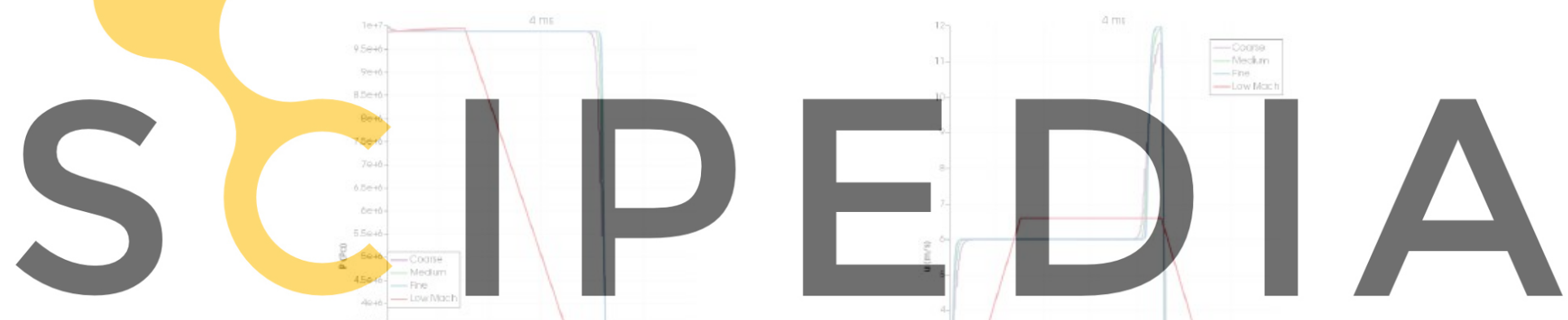

Register for free at https//www.scipedia.com to download the version without the watermark
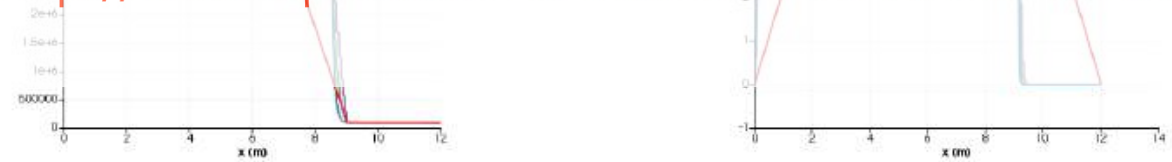

Figure 4: Strong bubble expansion. Compressible flow solution vs reference low-Mach number solution at $\mathrm{t}=4$ ms. Left: pressure distributions. Right: velocity distributions.

\subsection{Weak bubble expansion}

Figure 9 displays the time-evolution of the equilibrium pressure for the case of the weak bubble expansion (with an initial pressure level of $10 \mathrm{bar}$ in the bubble region). Because of the larger characteristic time associated with the physical phenomena in that case, there is no longer a visible time shift at the impact of the liquid column. The equilibrium pressure decreases with the mesh size (since numerical dissipation is reduced by the grid refinement) and better converges to its initial value with respect to the case of the strong bubble expansion. The agreement between the low-Mach and compressible solutions for the 

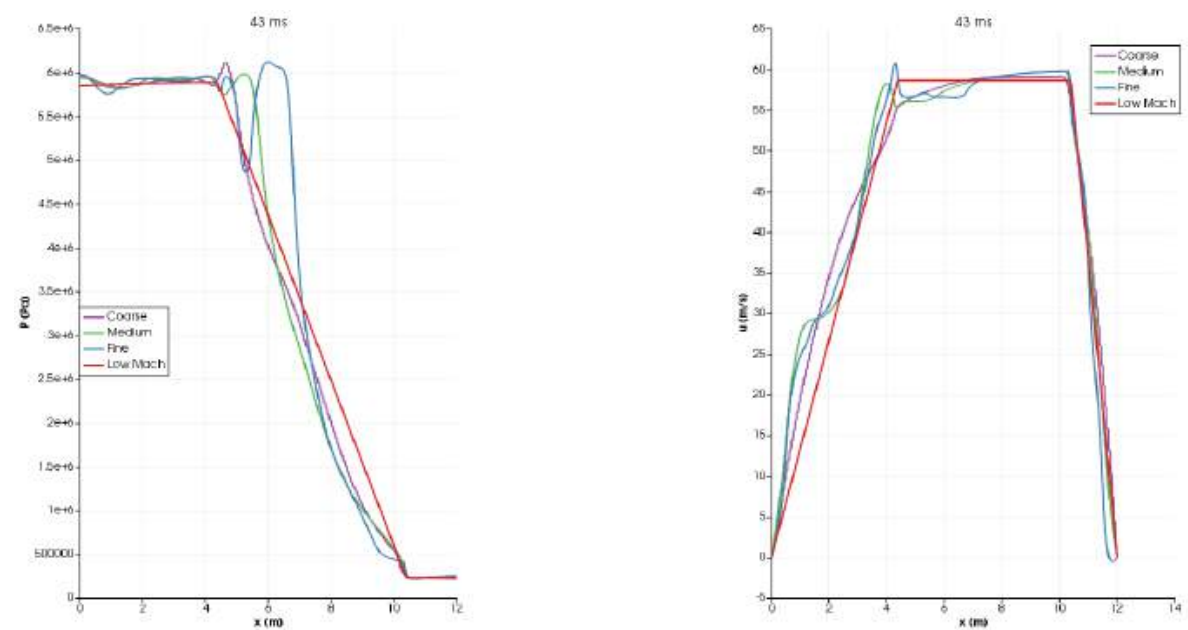

Figure 5: Strong bubble expansion. Compressible flow solution vs reference low-Mach number solution at $\mathrm{t}=42$ ms. Left: pressure distributions. Right: velocity distributions.
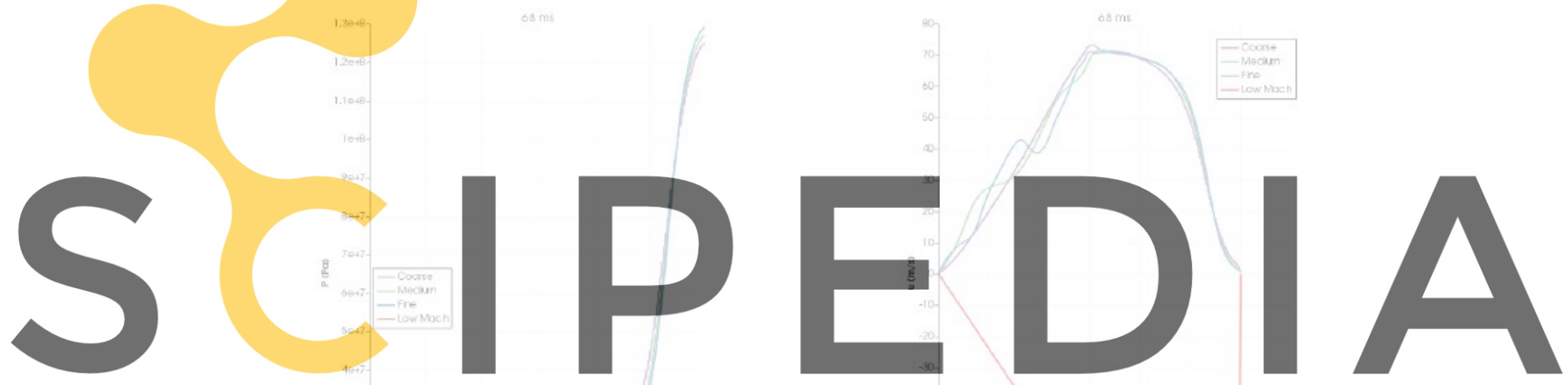

Register for free at https//www.scipedia.Com to download the version without the watermark

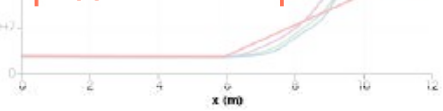

Figure 6: Strong bubble expansion. Compressible flow solution vs reference low-Mach number solution at $\mathrm{t}=68$ ms. Left: pressure distributions. Right: velocity distributions.

water kinetic energy remains very good over the whole computed time-interval.

\section{CONCLUSIONS AND PERSPECTIVES}

1D Bubble expansion problems at low-Mach number have been computed using a state-of-the art compressible method on a series of increasingly refined grids and the compressible numerical solutions have been compared with a reference low-Mach solution. The goal of this comparison is to assess whether the low-Mach solution is accurate enough to allow safety analysis for HCDA configurations. An accuracy criterion based on the exergy/anergy has been proposed for a thorough comparison of compressible and low-Mach solutions. It has been observed through numerical experiments that the low Mach number 

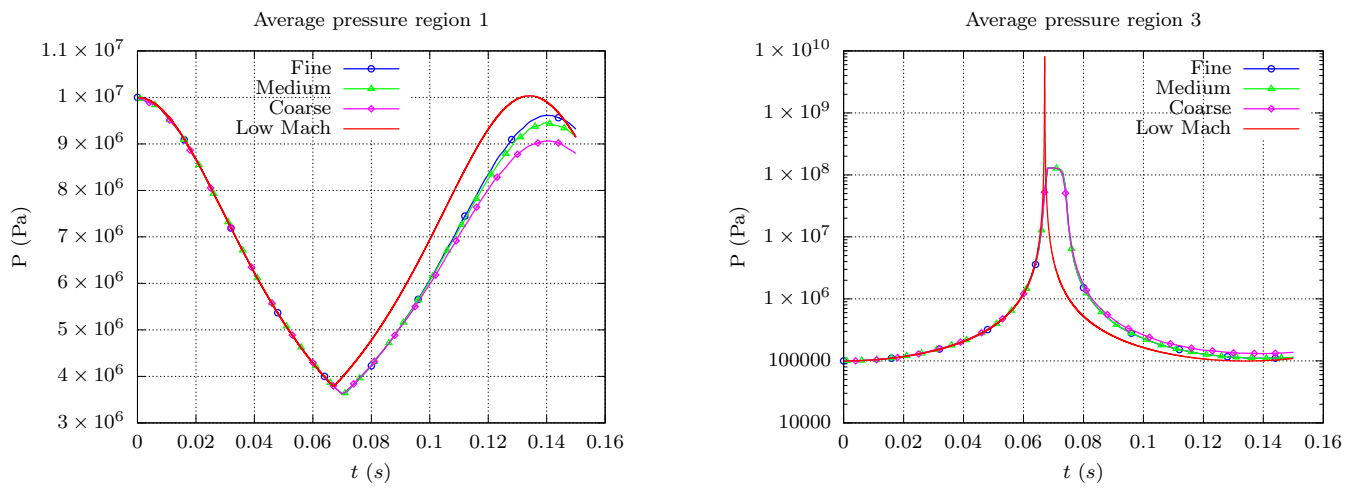

Figure 7: Strong bubble expansion. Comparison of the pressure time-evolution for the low-Mach solution and the compressible numerical solution using the increasingly refined grids. Left: time evolution of the average pressure in the bubble. Right: time-evolution of the average pressure in the absorber.

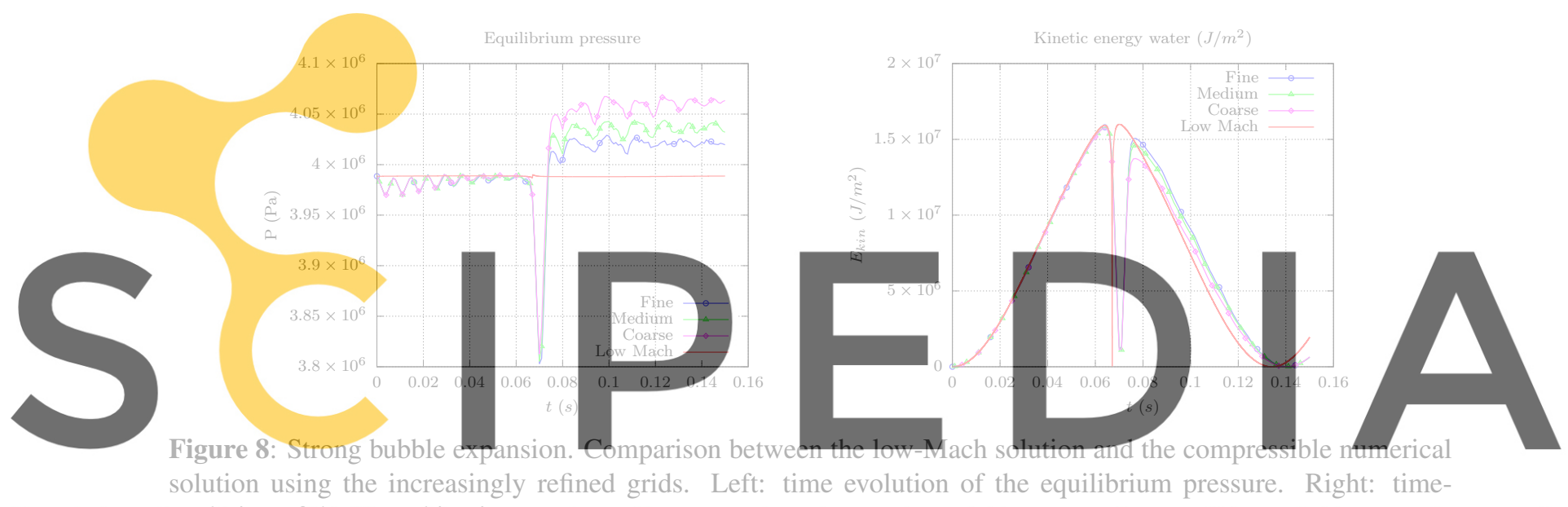

solution provides indeed a very good approximation of the grid-converged compressible solution for the weak expansion and an envelope solution for the strong expansion. This motivates the design of a lowMach number solver based on the splitting (1) which will offer a reduction of the CPU time consumption directly proportional with the ratio between the actual speed of sound $c$ used in the compressible simulations and the artificially reduced speed of sound $c_{A C}$ introduced in the Artificial Compressibility method.

\section{REFERENCES}

[1] Chang, Y.W.. Analysis of HCDA. Nuclear Engineering and Design 69 (1982) 345-358.

[2] Acker, D., Benuzzi, A., Yerkess, A., Louvet, J., MARA 01/02-experimental validation of the SEURBNUK and SIRIUS containment codes. In: Proceedings of the Sixth International Conference on Structural Mechanics in Reactor Technology (1981)

[3] Louvet, J., Hamon, P., Smith, B.L., Zucchini, A., MARA 10: an integral model experiment in support of LMFBR containment analysis. In: Proceedings of the Ninth International Conference 

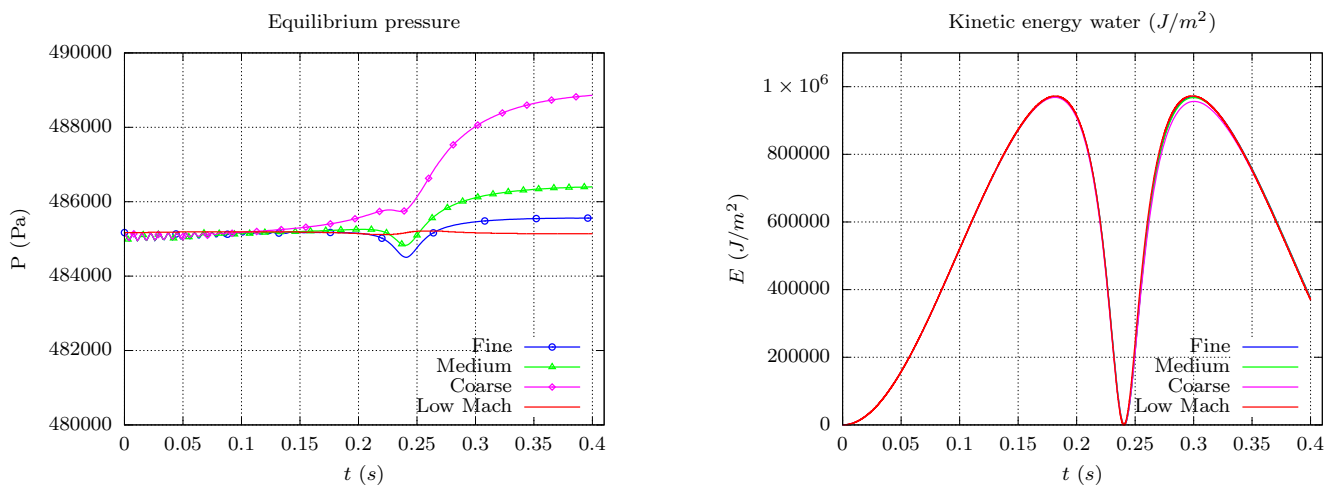

Figure 9: Strong bubble expansion. Comparison between the low-Mach solution and the compressible numerical solution using the increasingly refined grids. Left: time evolution of the equilibrium pressure. Right: timeevolution of the water kinetic energy.

on Structural Mechanics in Reactor Integrity, vol. E, Lausanne, Switzerland (1981), pp. 331-337.

[4] Fiche, C., Louvet, J., Smith, B.L., Zucchini, A., Theo- retical experimental study of flexible roof effects in an HCDA's simulation. In: Proceedings of the 8th International Conference on Structural Mechanics in Reactor Technology, Paper E 4/5, Brussels, Belgium, pp. 139-144 (1985).

[5] Robbe, M.F., Casadei, F., Comparison of various models for the simulation of a Core Disruptive Accident in the MARA 10 mock-up. Nuclear Engineering and Design 232 (2004) 301-326.

[6] Robbe, M.F., Lepareux, M., C ulating a Hypothetical Cor $119-158$.

[7] Robbe, M.F., Lepareux, M., Treille, E., Cariou
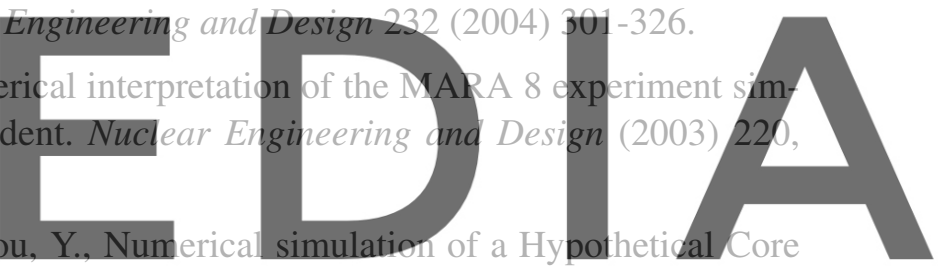

Disruptive Accident in a simple scale model of a nuclear reactor. Nuclear Engineering Design

Register for freenat feftpsy 15 wwwescipedia.com to download the version without the watermark

[8] Dellacherie, S., Jung, J., Omnes, P., and Raviart, P.-A., Construction of modified Godunov-type schemes accurate at any Mach number for the compressible Euler system. Mathematical Models and Methods in Applied Sciences (2016) 26 2525-2615.

[9] Beccantini, A., Cong-Huan, P., and Corre, C., Bubble expansion computations at low-Mach number regime. In preparation for submission to Journal of Computational Physics.

[10] Abgrall, R. and Saurel, R., Discrete equations for physical and numerical compressible multiphase mixtures. Journal of Computational Physics (2003) 186 361-396.

[11] Szargut, J., and Morris, D. R., Cumulative exergy consumption and cumulative degree of perfection of chemical processes. International Journal of Energy Research (1987) 11(2) 245-261. 